\title{
Спиновые состояния электронов в двойной квантовой точке в двумерном топологическом изоляторе со спин-орбитальным взаимодействием
}

\author{
(C) A.A. Суханов, В.А. Сабликов \\ Фрязинский филиал Института радиотехники и электроники им. В.А. Котельникова Российской академии наук, \\ 141190 Фрязино, Россия \\ E-mail: AASukhanov@yandex.ru
}

Поступила в Редакцию 24 апреля 2019 г.

В окончательной редакции 29 апреля 2019 г.

Принята к публикации 29 апреля 2019 г.

Изучены спектры и спиновая структура состояний двух взаимодействующих электронов, локализованных в двойной квантовой точке в двумерном топологическом изоляторе при наличии спин-орбитального взаимодействия. Найдено, что в такой системе может быть реализован синглет-триплетный переход в основном состоянии без магнитного поля. Спин-орбитальное взаимодействие приводит к расщеплению поляризованных триплетных уровней и к антикроссингу при пересечении одного из них с синглетом.

Ключевые слова: топологические изоляторы, спин-орбитальное взаимодействие, синглет-триплетный переход, квантовая точка.

DOI: 10.21883/FTP.2019.09.48134.17

\section{1. Введение}

Вопрос о спине основного состояния взаимодействующих электронов представляет собой фундаментальную проблему в квантовой физике небольшого числа частиц, имеющую большое значение для создания спинполяризованных состояний и для реализации спиновых кубитов на квантовых точках [1,2]. Спиновое состояние локализованных электронов определяется балансом зависящих от спина компонентов энергии системы, к которым прежде всего относится обменное взаимодействие, но и не только оно, так как прямое взаимодействие, взаимодействие с потенциалом, формирующим связанные состояния, и кинетическая энергия также зависят от спина. Наибольший интерес вызывает модельная система двух электронов. В этом случае имеется четкий результат, который связывают с именем Вигнера. Согласно теореме Вигнера, основное состояние является синглетом при любом парном взаимодействии, не зависящем от спина [3]. Поэтому синглет-триплетный переход возможен только при наличии магнитного поля. Другой не менее известный классический результат относится к системе достаточно большого количества электронов. Это полуэмпирическое правило Хунда $[4,5]$, согласно которому наименьшей энергией обладает состояние с наибольшим спином. Вопрос о том, при каком количестве электронов это правило применимо, не имеет определенного ответа, но известно, что в случае двух электронов оно несправедливо. Тем не менее триплетное состояние все же удается сделать основным, если двухэлектронная система связана с близко расположенной дополнительной квантовой точкой, содержащей большое количество электронов. Электроны в большой квантовой точке служат посредниками, благодаря которым увеличивается обменное взаимодействие рассматриваемых электронов [6,7].
Такие выводы сделаны для систем с обычным спектром. Однако применительно к современным топологически нетривиальным электронным системам проблема спина основного состояния и синглет-триплетных переходов мало исследована. Мы изучили ее для двух электронов в системах с двухзонным спектром в топологической и тривиальной фазах в двумерных (2D) системах [8]. Было установлено, что в топологической фазе теорема Вигнера при определенных условиях нарушается и основное состояние двух электронов может стать поляризованным триплетом.

В настоящей работе изучены эффекты, обусловленные спин-орбитальным взаимодействием (СОВ), связанным с пространственной асимметрией, в двойной квантовой яме в 2D топологическом изоляторе (ТИ). Показано, что СОВ приводит к значительной перестройке триплетных состояний, но при несильном СОВ сохраняются четко выраженные переходы между измененными синглетным и триплетными состояниями. Интересно, что поляризованные триплеты расщепляются на два состояния и соответственно имеются два таких перехода, причем антикроссинг происходит только при пересечении одного из триплетных уровней с синглетом. Исследована спиновая структура состояний в изолированной квантовой яме и в двухъямной структуре и обсуждена природа синглеттриплетного перехода.

\section{2. Модель двойной квантовой ямы}

Электроны в двойной квантовой яме в 2D ТИ описываются гамильтонианом

$$
H=H_{\mathrm{TI}}+V_{A}\left(\left|\mathbf{r}-\mathbf{R}_{A}\right|\right)+V_{B}\left(\left|\mathbf{r}-\mathbf{R}_{B}\right|\right),
$$

где $H_{\mathrm{TI}}$ - гамильтониан электрона в ТИ, который мы представим в виде суммы стандартного гамильтониана 
$H_{\mathrm{BHZ}}$ модели $\mathrm{BHZ}[9]$ и гамильтониана $\mathrm{COB} H_{\mathrm{SOI}}$ :

$$
H_{\mathrm{TI}}=H_{\mathrm{BHZ}}+H_{\mathrm{SOI}}
$$

Гамильтониан модели $\mathrm{BHZ}$ в безразмерной форме имеет вид

$$
H_{\mathrm{BHZ}}=\left(\begin{array}{cc}
h(\hat{k}) & 0 \\
0 & h^{*}(-\hat{k})
\end{array}\right),
$$

где $h(\hat{k})=\left(\mu-\hat{k}^{2}\right) \hat{\tau}_{z}+a\left(\hat{k}_{x} \hat{\tau}_{x}+\hat{k}_{y} \hat{\tau}_{y}\right), \hat{\tau}_{i}-$ матрицы Паули для псевдоспина, энергия нормирована на массовый член в гамильтониане $|M|$, а импульс на $\sqrt{|M / B|}$, $\mu=M /|M|, a=A / \sqrt{|M B|}$. Здесь величины $A, M$ и $B-$ параметры модели BHZ [9]. Параметр $\mu$ определяет топологическую $(\mu=-1)$ и тривиальную $(\mu=1)$ фазы.

В этой работе мы приняли во внимание $\mathrm{COB}$, обусловленное пространственной асимметрией с учетом асимметрии как в объеме [10], так и на интерфейсе [11]. Оба вида СОВ приводят к качественно близким результатам в спектрах, но образующиеся спиновые текстуры различаются направлением спинов. В первом случае гамильтониан COB записывается в виде

$$
H_{\mathrm{SOI}}=\Delta \hat{\tau}_{y} \oplus \hat{\sigma}_{y}
$$

где $\Delta-$ постоянная СОВ.

Потенциалы квантовых ям $V_{A}$ и $V_{B}$ для простоты будем считать одинаковыми, $V_{A}=V_{B}=V$, а векторами $\mathbf{R}_{A}$ и $\mathbf{R}_{B}$ обозначим положение ям.

Состояния двух взаимодействующих электронов описываются гамильтонианом

$$
H(1,2)=H(1) \oplus H(2)+U(1,2) I_{16},
$$

где $H(1)$ и $H(2)$ - приведенные выше одночастичные гамильтонианы электронов, обозначенных 1 и 2 , $U(1,2)$ - потенциал парного взаимодействия, $I_{16}-$ единичная матрица. Двухчастичная волновая функция описывается спинором 16 ранга.

Для нахождения спектра и волновых функций предположим, что расстояние $d$ между квантовыми ямами достаточно велико, так чтобы конфигурационное расщепление уровней квантовых ям, обусловленное туннелированием электронов между потенциальными ямами, и электрон-электронное взаимодействие можно было учесть по теории возмущений. При этом в качестве базисных функций возьмем двухчастичные волновые функции, составленные как антисимметризованные произведения одночастичных волновых функций гамильтониана изолированных квантовых ям $(A$ и $B)$.

Эти одночастичные волновые функции определяются гамильтонианом

$$
H_{0}=H_{\mathrm{TI}}+V\left(\left|\mathbf{r}-\mathbf{R}_{A, B}\right|\right)
$$

в котором $H_{\mathrm{TI}}$ - гамильтониан модели $\mathrm{BHZ}$ с включенным в него СОВ (см. уравнение (2)) и $V$ - потенциал изолированных квантовых ям.
Уравнение Шредингера с гамильтонианом (6) решается по методике, представленной нами в работах $[12,13]$. Основным в этой методике является допущение, что потенциал квантовой точки сильно локализован, так что волновая функция в области локализации потенциала изменяется мало. В результате удается аналитически найти волновые функции электрона локализованного в яме. Уровни энергии и волновые функции определяются безразмерными параметрами $v, \Lambda$ и $\delta /|M|$.

Параметр $v=|B|^{-1} \int V(r) d^{2} \mathbf{r}$ - определяет амплитуду потенциала квантовой ямы, а $\Lambda=\sqrt{|M / B|} / l-$ отношение характерной длины в модели BHZ к ширине $l$ области локализации потенциала. Кроме того энергия состояний зависит от параметра гибридизации электронной и дырочной зон $(a)$.

Так же как и в случае отсутствия $\mathrm{COB}$, при каждом знаке потенциала квантовой ямы в 2D ТИ могут возникать два состояния - одно электронного, а другое дырочного типов - с разными энергиями. Каждое из этих состояний двукратно вырождено, так как в силу симметрии обращения времени каждое такое состояние представляет крамерсову пару. Таким образом, одночастичные волновые функции представляются четырехкомпонентными спинорами, которые характеризуются индексом $e$ или $h$, обозначающим электронный или дырочный тип состояния, и индексом $\alpha$ или $\beta$, обозначающим состояние крамерсовой пары.

Несложный анализ и сравнение с численными расчетами показывают, что теория возмущений при расчете туннельного расщепления уровней квантовых ям справедлива при достаточно большом расстоянии между ямами, $d>6 \sqrt{|B / M|}$ в широком диапазоне значений параметра СОВ $\Delta$.

Двухчастичные базисные функции нулевого приближения, на которых строится теория возмущений для электрон-электронного взаимодействия, формируются одночастичными волновыми функциями $\Psi_{n \gamma}(\mathbf{r})$, найденными по теории возмущений для двух туннельным образом связанных ям

$$
\begin{aligned}
\Psi_{n \gamma, m \gamma^{\prime}}^{(0)} & (1,2)=\frac{1}{\sqrt{2}} \\
& \times\left[\Psi_{n \gamma}(1) \otimes \Psi_{m \gamma^{\prime}}(2)-\Psi_{m \gamma^{\prime}}(1) \otimes \Psi_{n \gamma}(2)\right] .
\end{aligned}
$$

Здесь индекс $n(m)$ обозначает симметричные $(s)$ или антисимметричные $(a)$ двухъямные волновые функции для состояний электронного или дырочного типов, а $\gamma, \gamma^{\prime}=(\alpha, \beta)$ - индексы состояний крамерсовой пары.

Дальнейшее упрощение задачи возможно, если мы ограничимся рассмотрением всего одной пары одночастичных уровней, образовавшейся при туннельном расщеплении одного уровня в изолированной квантовой яме. Такое упрощение оправдано, если квантовая яма достаточно узкая и расстояние между ямами достаточно велико. Условия для этих упрощений обсуждены в работе [8] и соответствуют приведенному выше неравенству $d>6 \sqrt{|B / M|}$. 
В этом случае одночастичные состояния в связанных ямах классифицируются только как симметричные (связывающие) и антисимметричные (антисвязывающие), которые мы обозначим индексами $s$ и $a$ соответственно. В результате имеем шесть базисных двухчастичных волновых функций

$$
\left(\Psi_{s \alpha, s \beta}^{(0)}, \Psi_{s \alpha, a \beta)}^{(0)}, \Psi_{a \alpha, s \beta}^{(0)}, \Psi_{s \alpha, a \alpha}^{(0)}, \Psi_{s \beta, a \beta}^{(0)}, \Psi_{a \alpha, a \beta}^{(0)}\right)^{T} .
$$

Далее для простоты они нумеруются одним индексом $j=(1,6)$.

Волновая функция гамильтониана (5) представляется в виде

$$
\Psi(1,2)=\sum_{j=1}^{6} C_{j} \Psi_{j}^{(0)}(1,2) .
$$

Используя уравнения (5) и (9), нетрудно получить однородную систему уравнений для коэффициентов $C_{j}$, детерминант которой определяет спектр. Матрицу детерминанта и матричные коэффициенты мы не приводим из-за того, что они слишком громоздки. Результаты расчета приведены далее.

\section{3. Спектр и синглет-триплетный переход}

В топологической фазе 2D ТИ короткодействующий потенциал $V(r)$ создает два уровня при любом знаке потенциала в отличие от тривиальной фазы, когда в рамках модели BHZ имеется только один уровень для потенциала любого знака $[12,13]$.

Локализованные состояния, соответствующие этим уровням, классифицируются как электроноподобные и дырочноподобные. Они существенно различаются распределением электронной плотности и плотности компонент спинорной волновой функции. В двойной квантовой яме эти уровни расщепляются на два вследствие конфигурационного взаимодействия ям. Мы рассматриваем каждую пару образовавшихся уровней отдельно, полагая, что они достаточно разнесены по энергии, а расщепление каждой пары, напротив, мало. Свойства двухчастичных спектров, порождаемые каждым из этих уровней, существенно отличаются. Так, при положительном потенциале синглет-триплетный переход возможен только для дырочноподобных состояний [8]. Поэтому только они и будут рассмотрены далее.

Энергетический спектр имеет 6 уровней $\varepsilon_{j}$. Мы изучили их зависимость от амплитуды потенциала электронэлектронного взаимодействия $u$, нормированного как и потенциал ям на $|M|$, и выяснили, какие эффекты возникают благодаря СОВ и к чему приводит совместное действие СОВ и электрон-электронного взаимодействия. На рис. 1 представлен спектр, рассчитанный для $\mathrm{COB}$, обусловленного объемной асимметрией [10], с параметром $\Delta=0.2$, определенным как в работе [10] и нормированным на $|M|$.

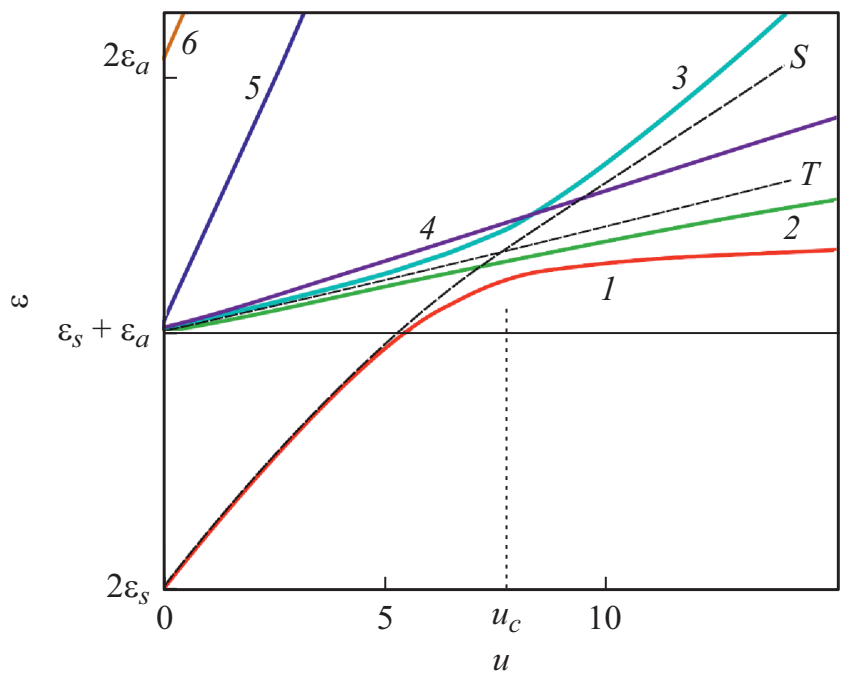

Рис. 1. Двухчастичный спектр электронов в двойной симметричной квантовой яме при наличии СОВ. Расчеты проведены для параметров $v=20, \Lambda=3, a=2, \Delta=0.2, d=8$.

В задаче имеются три характерные энергии: $2 \varepsilon_{s}$, $\varepsilon_{a}+\varepsilon_{s}$ и $2 \varepsilon_{a}$, которые определяют спектр при отсутствии СОВ и электрон-электронного взаимодействия. Здесь $\varepsilon_{s}$ и $\varepsilon_{a}$ - энергии симметричного и антисимметричного одночастичных состояний. В этом предельном случае уровни классифицируются обычным образом: линия 1 - синглет $|s \uparrow s \downarrow\rangle ; 2$ и 3 поляризованные триплеты $|s \uparrow a \uparrow\rangle$ и $|s \downarrow a \downarrow\rangle ; 4-$ неполяризованный триплет, составленный из состояний $|s \uparrow a \downarrow\rangle$ и $|s \downarrow a \uparrow\rangle ; 5$ - синглет, составленный из состояний $|s \uparrow a \downarrow\rangle$ и $|s \downarrow a \uparrow\rangle ; 6$ - синглет $|a \uparrow a \uparrow\rangle$. В связи с такой привычной терминологией необходимо заметить, что в рассматриваемом случае она не вполне точна. Дело в том, что в модели BHZ не сохраняется полный спин, так как оператор $S^{2}$ не коммутирует с гамильтонианом. Определенное значение имеет только $S_{z}$. Поэтому при ненулевом взаимодействии между электронами нет трехкратно вырожденного по спину состояния. Имеется только двукратно вырожденное, которое часто тоже называют триплетом.

При увеличении амплитуды взаимодействия $u$ в отсутствие СОВ происходит смешивание синглетных состояний $|s \uparrow s \downarrow\rangle$ и $|a \uparrow a \downarrow\rangle$, состояния $|s \uparrow a \uparrow\rangle$ и $|s \downarrow a \downarrow\rangle$ остаются вырожденными, а также изменяются составы в смеси состояний $|s \uparrow a \downarrow\rangle$ и $|s \downarrow a \uparrow\rangle$, образующих неполяризованный триплет и синглет. При этом синглет $|s \uparrow s \downarrow\rangle$, обозначенный линией 1 , пересекает триплеты (2 и 3), как это было показано ранее [8]. Поведение этих термов показано пунктирами $S$ и $T$ на рис. 1 . Нетривиальная особенность состоит в том, что при критическом значении $u=u_{c}$ происходит пересечение синглетного и триплетного термов. При $u>u_{c}$ триплетное состояние становится основным и, таким образом, теорема Вигнера нарушается. Заметим, что такое поведение термов 
возможно только при инверсии зонного спектра, т.е. только в топологической фазе, причем при небольших значениях параметра гибридизации $a(a<10)$, когда система достаточно далека от точки топологического перехода.

Критическая для синглет-триплетного перехода величина $u_{c}$ зависит от потенциала $v$ квантовых ям, так что пересечение термов и синглет-триплетный переход могут быть реализованы путем изменения потенциала $v$.

Эффект СОВ состоит прежде всего в том, что дважды вырожденный триплетный уровень (пунктир $T$ ) расщепляется на два уровня (кривые 2 и 3). Вблизи точки синглет-триплетного перехода происходит расталкивание (антикроссинг) синглетного уровня и верхнего уровня расщепленного триплета.

Интересно, что все проекции спиновой плотности $S_{i}$ в состояниях, образовавшихся при расщеплении дважды вырожденного триплетного уровня (линии 2 и 3 на рис. 1), равны нулю: $\Psi_{j=2,3}^{+}(\mathbf{r}) S_{i} \Psi_{j=2,3}(\mathbf{r})=0$, т. е. образовавшиеся двухэлектронные состояния не поляризованы.

\section{4. О механизме перестройки основного состояния}

Ответ на вопрос о том, какое состояние двух электронов - с параллельными или противоположными спинами - энергетически выгоднее, определяется соотношением между зависящими от спина компонентами энергии, к которым в рассматриваемой системе прежде всего относится обменная энергия и энергия прямого кулоновского взаимодействия. Важны также и энергия взаимодействия электрона с зарядами, формирующими квантовую яму, и кинетическая энергия, но в случае квантовых ям с сильно локализованным потенциалом, находящихся на достаточно большом расстоянии друг от друга, эти последние компоненты не так существенны.

Зависимость энергии взаимодействия электронов от спина связана с наличием нескольких компонент спинора, описывающего данное состояние, которые имеют различные пространственные распределения для разных спиновых конфигураций. В обычной ситуации, для которой установлена теорема Вигнера, рассматриваются электроны в системе с однозонным спектром и состояния описываются спинором второго ранга. В этом случае спектр эволюционирует при увеличении амплитуды потенциала электрон-электронного взаимодействия следующим образом. Энергия нижнего синглета возрастает из-за увеличения энергии кулоновского отталкивания. Энергия поляризованных триплетов растет значительно медленнее из-за того, что энергия отталкивания в значительной мере компенсируется обменным взаимодействием. Однако с ростом межэлектронного взаимодействия обменная энергия уменьшается и в пределе $u \rightarrow \infty$ перестает играть роль, так что синглетный и триплетный термы асимптотически сближаются, но не пересекаются [14].

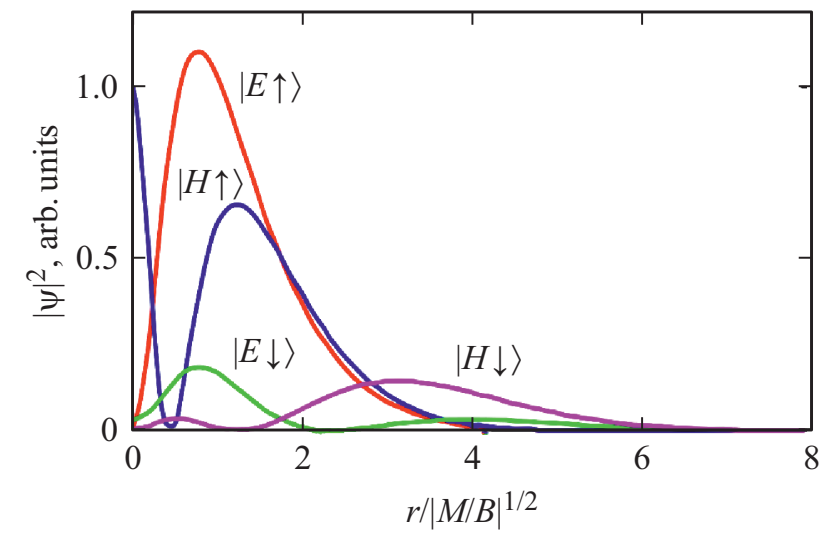

Рис. 2. Радиальное распределение компонент спинора дырочноподобного состояния электрона, локализованного в изолированной квантовой яме. Для компонент со спином $(\downarrow)$ масштаб увеличен в 4 раза. Расчеты проведены для параметров: $a=2, \Lambda=4, \Delta=0.2, v=40$. Энергия связанного состояния $\varepsilon=0.3289$.

В изучаемой нами системе одночастичные волновые функции имеют четыре компоненты, соответствующие двум направлениям спина и двум базисным орбиталям. В модели BHZ базис образуется состояниями электронной и дырочной зон с разными угловыми моментами: $\{|E, 1 / 2\rangle,|H, 3 / 2\rangle,|E,-1 / 2\rangle,|H,-3 / 2\rangle\}$. Энергии прямого $W_{C}$ и обменного $W_{\mathrm{ex}}$ взаимодействий электронов, описываемых волновыми функциями $\Psi\left(\mathbf{r}_{1}\right)=\left(\psi_{1}, \psi_{2}, \psi_{3}, \psi_{4}\right)^{T}$ и $\Phi\left(\mathbf{r}_{2}\right)=\left(\phi_{1}, \phi_{2}, \phi_{3}, \phi_{4}\right)^{T}$, имеют вид

$$
\begin{aligned}
& W_{C}=\iint d^{2} \mathbf{r}_{1} d^{2} \mathbf{r}_{2} \Psi^{+}\left(\mathbf{r}_{1}\right) \Psi\left(\mathbf{r}_{1}\right) U\left(\left|\mathbf{r}_{1}-\mathbf{r}_{2}\right|\right) \Phi^{+}\left(\mathbf{r}_{2}\right) \Phi\left(\mathbf{r}_{2}\right), \\
& W_{\mathrm{ex}}=\iint d^{2} \mathbf{r}_{1} d^{2} \mathbf{r}_{2} \Psi^{+}\left(\mathbf{r}_{1}\right) \Phi\left(\mathbf{r}_{1}\right) U\left(\left|\mathbf{r}_{1}-\mathbf{r}_{2}\right|\right) \Phi^{+}\left(\mathbf{r}_{2}\right) \Psi\left(\mathbf{r}_{2}\right)
\end{aligned}
$$

Очевидно, что наличие псевдоспиновых компонент существенно изменяет величины как прямого, так и обменного взаимодействия и их зависимость от спинов, особенно если учесть, что величина обменных интегралов определяется не только спиновыми, но и псевдоспиновыми компонентами.

Вклад компонентов спинора волновой функции в зарядовую и спиновую плотности, которые обычно ассоциируются с прямым и обменным взаимодействием, проще всего продемонстрировать на примере связанного состояния в случае одной изолированной квантовой ямы. На рис. 2 приведено радиальное распределение компонентов $\left|\psi_{i=(1,4)}(\mathbf{r})\right|^{2}$ для связанного состояния дырочного типа. Распределение спиновой плотности приведено на рис. 3. Компонента спиновой плотности $S_{z}$ (рис. 3,a), нормальная к плоскости системы, изменяется с расстоянием немонотонно и при удалении от центра даже изменяет знак. Тангенциальная компонента намного меньше по величине, но она все же имеется и изменяется с 

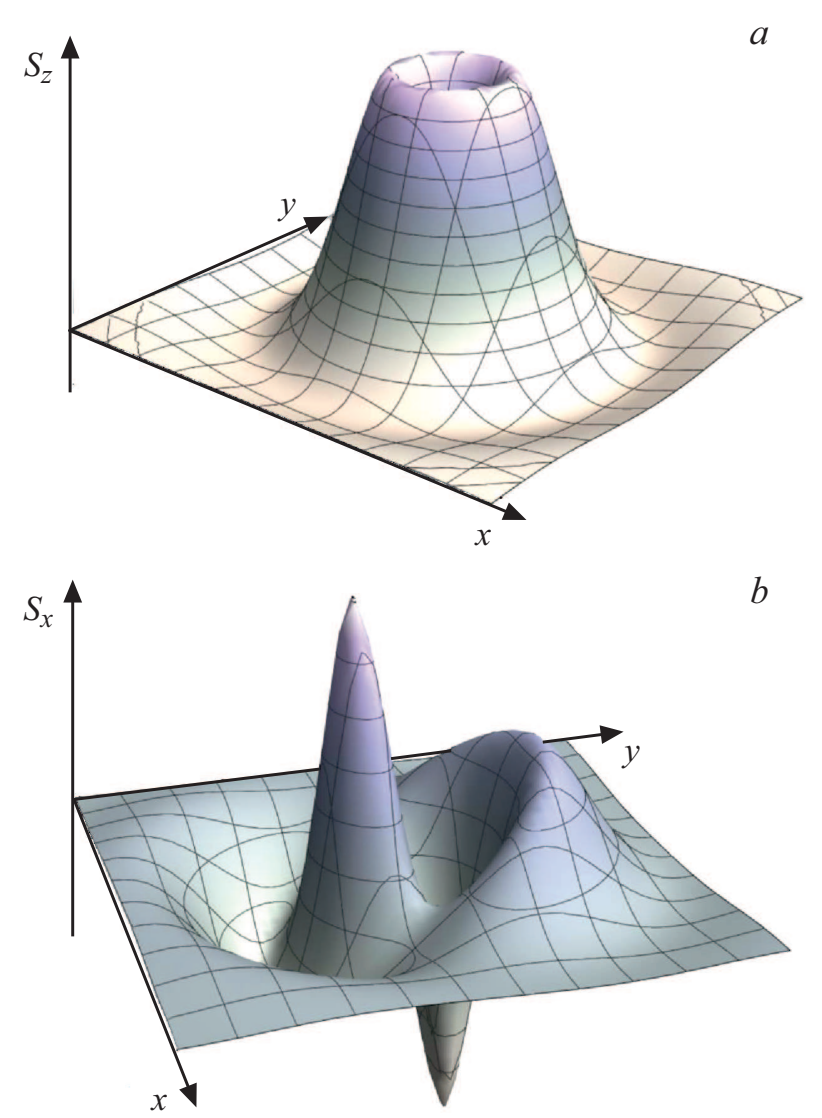

Рис. 3. Распределение $S_{z}(a)$ и $S_{x}(b)$ компонент спиновой плотности электрона, локализованного в изолированной квантовой яме, в дырочноподобном состоянии. Для компоненты $S_{x}$ масштаб увеличен в 20 раз. Использованы те же параметры, как и на рис. 2.

расстоянием совершенно необычным образом, как это показано на рис. $3, b$ для компоненты $S_{x}$.

Очевидно, что необычные по сравнению с тривиальной системой распределения плотности заряда и плотности спина могут привести к значительному изменению величин как прямого, так и обменного взаимодействий электронов в двух ямах, с чем и связано нарушение теоремы Вигнера.

\section{5. Заключение}

Проведенные нами расчеты показывают, что с ростом амплитуды электрон-электронного взаимодействия синглетный и триплетный термы при определенных условиях пересекаются, если СОВ отсутствует. При слабом СОВ происходит расщепление триплета. При этом один из расщепленных уровней пересекается с синглетом, тогда как для другого имеет место антикроссинг. Формально в расчетах, проведенных в настоящей статье по теории возмущений, из-за наличия псевдоспиновых компонент спинора обменное взаимодействие описывается тремя матричными элементами в отсутствие СОВ и шестью при его наличии, а прямое взаимодействие в обоих случаях - только тремя. Все вместе они и определяют энергии разных спиновых конфигураций.

\section{Финансирование работы}

Работа выполнена в рамках государственного задания ИРЭ РАН и при поддержке Программы Президиума РАН № 13 „Основы высоких технологий и использование особенностей наноструктур в науках о природе “.

\section{Конфликт интересов}

Авторы заявляют, что у них нет конфликта интересов.

\section{Список литературы}

[1] C. Kloeffel, D. Loss. Ann. Rev. Condens. Matter Phys., 4, 51 (2013).

[2] D.D. Awschalom, L.C. Bassett, A.S. Dzurak, E.L. Hu, J.R. Petta. Science, 339, 1174 (2013).

[3] E. Lieb, D. Mattis. Phys. Rev., 125, 164 (1962).

[4] R.J. Boyd. Nature, 310, 480 (1984).

[5] W. Kutzelnigg, J.D. Morgan III. Z. Phys. D, 36, 197 (1996).

[6] F.K. Malinowski, F. Martins, T.B. Smith, S.D. Bartlett, A.C. Doherty, P.D. Nissen, S. Fallahi, G.C. Gardner, M.J. Manfra, C.M. Marcus, F. Kuemmeth. Phys. Rev. X, 8, 011045 (2018).

[7] K. Deng, F.A. Calderon-Vargas, N.J. Mayhall, E. Barnes. Phys. Rev. B, 97, 245301 (2018).

[8] V.A. Sablikov, A.A. Sukhanov. Phys. Rev. B, 98, 115423 (2018).

[9] B.A. Bernevig, T.L. Hughes, S.-C. Zhang. Science, 314, 1757 (2006).

[10] M. König, H. Buhmann, L.W. Molenkamp, T. Hughes, C.X. Liu, X.-L. Qi, S.-C. Zhang. J. Phys. Soc. Jpn., 77, 031007 (2008).

[11] S.A. Tarasenko, M.V. Durnev, M.O. Nestoklon, E.L. Ivchenko, J.-W. Luo, A. Zunger. Phys. Rev. B, 91, 081302 (2015).

[12] V.A. Sablikov, A.A. Sukhanov. Phys. Status Solidi RRL, 8, 853 (2014).

[13] V.A. Sablikov, A.A. Sukhanov. Phys. Rev. B, 91, 075412 (2015).

[14] Y. Gindikin, V.A. Sablikov. J. Phys.: Condens. Matter, 23 (17), 175601 (2011).

Редактор А.Н. Смирнов 


\title{
Spin states of electrons in a double quantum dot in a two-dimensional topological insulator with spin-orbit interaction
}

\author{
A.A. Sukhanov, V.A. Sablikov \\ Fryazinsky Branch of Kotelnikov Institute \\ of Radioengineering and Electronics, \\ Russian Academy of Sciences, \\ 141190 Fryazino, Russia
}

Abstract We study spectra and spin structure of quantum states of two interacting electrons localized in a double quantum dot in a two-dimensional topological insulator in the presence of spinorbit interaction. It was found that in such a system a singlet-triplet transition in the ground state can be realized without any magnetic field. The spin-orbit interaction leads to the splitting of polarized triplet levels and to the avoided crossing of the singlet level and one of triplets. 